\title{
Effect of short-term $\varepsilon$-aminocaproic acid treatment on patients undergoing endovascular coil embolization following aneurysmal subarachnoid hemorrhage
}

\author{
Mahdi Malekpour, MD,, Charles Kulwin, MD, ${ }^{1}$ Bradley N. Bohnstedt, MD, ${ }^{1}$ Golnar Radmand, MSc, ${ }^{2}$ \\ Rishabh Sethia, BS, ${ }^{1}$ Stephen K. Mendenhall, MD,1 Jonathan Weyhenmeyer, MD, ${ }^{1}$ \\ Benjamin K. Hendricks, MD, ${ }^{1}$ Thomas Leipzig, MD, ${ }^{1}$ Troy D. Payner, MD, ${ }^{1}$ Mitesh V. Shah, MD, ${ }^{1}$ \\ John Scott, MD, ${ }^{1}$ Andrew DeNardo, MD, ${ }^{1}$ Daniel Sahlein, MD, ${ }^{1}$ and Aaron A. Cohen-Gadol, MD, MSc ${ }^{1,3}$

\begin{abstract}
${ }^{1}$ Goodman Campbell Brain and Spine, Indiana University, Department of Neurological Surgery; ${ }^{3}$ Indiana University Simon Cancer Center, Indianapolis, Indiana; and 2Department of Biostatistics, Shahid Beheshti University of Medical Sciences, Tehran, Iran
\end{abstract}

OBJECTIVE Aneurysmal rebleeding before definitive obliteration of the aneurysm is a cause of mortality and morbidity. There are limited data on the role of short-term antifibrinolytic therapy among patients undergoing endovascular intervention.

METHODS All consecutive patients receiving endovascular therapy for their ruptured saccular aneurysm at the authors' institution between 2000 and 2011 were included in this study. These patients underwent endovascular coiling of their aneurysm within 72 hours of admission. In patients receiving $\varepsilon$-aminocaproic acid (EACA), the EACA administration was continued until the time of the endovascular procedure. Complications and clinical outcomes of endovascular treatment after aneurysmal subarachnoid hemorrhage (aSAH) were compared between EACA-treated and untreated patients.

RESULTS During the 12-year study period, 341 patients underwent endovascular coiling. Short-term EACA treatment was administered in 146 patients and was withheld in the other 195 patients. EACA treatment did not change the risk of preinterventional rebleeding in this study (OR 0.782, 95\% Cl 0.176-3.480; $p=0.747$ ). Moreover, EACA treatment did not increase the rate of thromboembolic events. On the other hand, patients who received EACA treatment had a significantly longer duration of hospital stay compared with their counterparts who were not treated with EACA (median 19 days, interquartile range [IQR] 12.5-30 days vs median 14 days, IQR 10-23 days; $p<0.001)$. EACA treatment was associated with increased odds of shunt requirement (OR 2.047, 95\% $\mathrm{Cl} 1.043-4.018 ; p=0.037$ ) and decreased odds of developing cardiac complications (OR $0.138,95 \% \mathrm{Cl} 0.031-0.604 ; p=0.009)$ and respiratory insufficiency $(\mathrm{OR} 0.471,95 \% \mathrm{Cl}$ $0.239-0.926 ; p=0.029$ ). Short-term EACA treatment did not affect the Glasgow Outcome Scale score at discharge, 6 months, or 1 year following discharge.

CONCLUSIONS In this study, short-term EACA treatment in patients who suffered from aSAH and received endovascular aneurysm repair did not decrease the risk of preinterventional rebleeding or increase the risk of thrombotic events. EACA did not affect outcome. Randomized clinical trials are required to provide robust clinical recommendation on shortterm use of EACA.

https://thejns.org/doi/abs/10.3171/2016.4.JNS152951

KEY WORDS cerebral aneurysm; subarachnoid hemorrhage; $\varepsilon$-aminocaproic acid; endovascular; outcome; complication; vascular disorders 
A NEURYSMAL subarachnoid hemorrhage (aSAH) is a devastating condition with a mortality rate of at least $25 \% .{ }^{6}$ Approximately $10 \%$ of patients with aSAH die before reaching the hospital. In general, the most important long-term prognostic factors following acute aSAH are the patient's age, quantity of subarachnoid blood detected on the initial CT scan, and clinical condition on admission..$^{9,22,23,38}$

The mortality risk for patients who reach the hospital is increased by aneurysmal rebleeding and subsequent arterial vasospasm resulting in cerebral ischemia. ${ }^{3,6}$ Current aSAH management focuses on early circulatory exclusion of the ruptured aneurysm and aggressive management of potential secondary complications., ${ }^{5,6}$ Management at centers with experienced neurovascular surgeons, endovascular interventionists, and neurointensive care units has led to a relative improvement in case fatalities over the last 2 decades. ${ }^{6,724}$

Patients with aSAH are at substantial risk of rebleeding, with rehemorrhage rates of $4 \%$ within 24 hours, $20 \%$ within 2 weeks, and 50\% within 6 months after the initial bleed. ${ }^{44}$ Aneurysm repair, therefore, should preferably be performed as soon as logistically possible, generally within 24-72 hours of presentation. ${ }^{6}$ Prior to treatment, antifibrinolytic agents such as $\varepsilon$-aminocaproic acid (EACA) have been used to prevent rebleeding. Current American Stroke Association guidelines state, "For patients with an unavoidable delay in obliteration of aneurysm, a significant risk of rebleeding, and no compelling medical contraindications, short-term ( $<72$ hours) therapy with tranexamic acid or aminocaproic acid is reasonable to reduce the risk of early aneurysm rebleeding."

We previously studied the effect of short-term EACA treatment on aSAH patients before early microsurgical intervention, but there are few data about the role of shortterm EACA therapy before endovascular treatment. . $9,30^{2}$ These data are particularly important in light of the higher baseline thrombotic risks associated with endovascular treatment compared with microsurgical repair ${ }^{32}$ and the additional potential risk of thrombotic complications posed by antifibrinolytic agents. In this study, we investigated the role of short-term EACA treatment before endovascular coiling of ruptured cerebral aneurysms.

\section{Methods}

After obtaining Indiana University Institutional Review Board approval, we collected data for all consecutive patients who were treated endovascularly for ruptured saccular cerebral aneurysms from January 2000 through December 2011 at our institution. The database did not capture time to intervention, so we retrospectively reviewed the patients' charts to calculate this time interval. We could not find the accurate intervention time for 27 patients, yet all patients received endovascular treatment within 72 hours from the time of hospital admission. Clinical outcomes were assessed by independent clinical nurses who did not consistently know about the treatment group assignment at the time of their evaluations.

These patients were divided into 2 groups: those receiving EACA before intervention and those not receiving
EACA. EACA use was based on the consistent preference of the individual vascular neurosurgeon who was on call at the time of the patient's admission. The surgeons who preferred the use of EACA and those who did not were equally and randomly assigned to taking neurovascular call. This fact is likely to introduce some degree of randomization for the administration of EACA. Treated patients received an intravenous loading dose of 5-10 g of EACA followed by a maintenance dose of $2 \mathrm{~g} / \mathrm{hr}$ until the time of their endovascular procedure.

Data related to demographics, aneurysm characteristics, complications, and outcomes of endovascularly treated aneurysms were retrospectively analyzed. Demographic data included age, race, sex, comorbidities, smoking status, Hunt and Hess score, and Fisher grade on admission., ${ }^{9,22,23}$ Aneurysm characteristics included size, location, and multiplicity. Complications were measured as rebleeding, vasospasm, clinical stroke, radiological infarction, hydrocephalus, need for shunt, cardiac complications, pulmonary edema, respiratory insufficiency, systemic complications, deep venous thrombosis, and death before hospital discharge. The length of hospital stay was also assessed.

Nonparametric variables are expressed as the median with interquartile range (IQR). Continuous variables were compared using the Student t-test and nonparametric variables, using the Mann-Whitney test. Chi-square and Fisher's exact tests were used to compare categorical variables. Multivariate logistic regression was used for assessing the adjusted effect of EACA treatment in the presence of variables that demonstrated a significant association with the use of EACA in the univariate analysis. The results are shown as odds ratios with $95 \%$ confidence intervals. Multivariate linear regression was used for assessing the relationship between EACA and length of stay as an outcome, adjusting the effect of aneurysm location, admission grade, and time to intervention.

Patients were evaluated through follow-up examinations at 6 and 12 months following discharge from the hospital. The overall survival analysis was performed using the Cox proportional hazards regression model. Probability values $<0.05$ are considered significant.

\section{Results}

During the 12-year study period, 341 aSAH patients received endovascular treatment at our institution. Tables 1 and 2 summarize patient characteristics for the studied population. As shown in Table 1, there was no statistically significant demographic difference between the patients who did or did not receive EACA treatment. Aneurysm characteristics (Table 2) differed between the 2 groups: the percentage of posterior circulation aneurysms was higher among the patients receiving EACA $(p=0.004)$. The distribution of the Hunt and Hess grade was also different between the 2 groups. Patients who underwent EACA treatment presented in a worse clinical grade $(\mathrm{p}=$ $0.039)$ and harbored more midline aneurysms $(\mathrm{p}=0.002)$. Finally, time to intervention was longer in patients receiving EACA ( $p<0.001)$. There was no other statistically significant difference in aneurysm characteristics between the groups. 
TABLE 1. Baseline characteristics of the studied patients*

\begin{tabular}{ccccc}
\hline Characteristic & $\begin{array}{c}\text { Total } \\
(\mathrm{n}=341)\end{array}$ & $\begin{array}{c}\text { No EACA } \\
(\mathrm{n}=195)\end{array}$ & $\begin{array}{c}\text { EACA } \\
(\mathrm{n}=146)\end{array}$ & $\begin{array}{c}\mathrm{p} \\
\text { Value }\end{array}$ \\
\hline $\begin{array}{c}\text { Mean age in } \\
\text { yrs } \pm \text { SD }\end{array}$ & $59.26 \pm 14.75$ & $60.27 \pm 14.91$ & $57.92 \pm 4.49$ & 0.147 \\
\hline $\begin{array}{c}\text { Sex } \\
\text { Female }\end{array}$ & $239(70.1)$ & $141(72.3)$ & $98(67.1)$ & \\
Male & $102(29.9)$ & $54(27.7)$ & $48(32.9)$ & \\
\hline Race & & & & 0.847 \\
White & $307(90.0)$ & $175(89.7)$ & $132(90.4)$ & \\
Black & $20(5.9)$ & $11(5.6)$ & $9(6.2)$ & \\
Hispanic & $7(2.1)$ & $5(2.6)$ & $2(1.4)$ & \\
Asian & $2(0.6)$ & $1(0.5)$ & $1(0.7)$ & \\
Other & $4(1.2)$ & $3(1.5)$ & $1(0.7)$ & \\
Unknown & $1(0.3)$ & $0(0)$ & $1(0.7)$ & \\
\hline
\end{tabular}

* Values are reported as the number of patients (\%) unless specified otherwise.

Twelve patients (3.5\%; 6 patients in each group) suffered from aneurysmal rebleeding before endovascular treatment (Table 3). Symptomatic vasospasm was detected in $17 \%$ of the patients, with a trend toward increased vasospasm in the EACA-treated versus untreated patients ( $\mathrm{p}$ $=0.072$ ); significantly more shunts were placed in the patients receiving EACA treatment $(\mathrm{p}=0.004)$. Patients receiving EACA required a longer duration of hospital stay than the untreated patients $(\mathrm{p}<0.001)$, possibly reflecting their higher Hunt and Hess grade at admission. Clinical stroke and radiographic infarct rates were not statistically different between the 2 groups. Death during hospitalization was not significantly different between the 2 groups in the univariate analysis $(\mathrm{p}=0.460)$.

Table 4 shows the multivariate analysis for complications and outcomes while accounting for the differences (location of the aneurysm, Hunt and Hess grade at presentation, and time to intervention) between the 2 groups. EACA treatment did not change the risk of preinterventional rebleeding in this study (OR $0.782,95 \%$ CI $0.176-3.480 ; p=0.747$ ). More shunts were placed in the group receiving EACA (OR 2.047, 95\% CI 1.043-4.018; p $=0.037$ ), but there were fewer cardiac complications (OR $0.138,95 \%$ CI $0.031-0.604 ; p=0.009$ ) and cases of respiratory insufficiency (OR $0.471,95 \%$ CI $0.239-0.926$; $p$ $=0.029)$ in this group. The adjusted odds of death in patients who received EACA were not significantly different from the untreated group (OR 1.152, 95\% CI 0.560-2.373; $p=0.700$ ). Multivariate analysis of the length of stay demonstrated that administration of the EACA increases the mean length of stay by 4.161 days (SD 1.499 days, $\mathrm{p}=$ 0.006).

Figure 1 shows the survival analysis performed using the Cox regression model. EACA treatment, aneurysm location, and Hunt and Hess grade at admission were independent variables in the model. The results of analysis show that EACA treatment had a nonsignificant impact on improving survival (adjusted HR 0.998, 95\% CI 0.5411.839; $\mathrm{p}=0.994)$.
TABLE 2. Comparison of aneurysm characteristics in the studied patients*

\begin{tabular}{|c|c|c|c|c|}
\hline $\begin{array}{c}\text { Aneurysm } \\
\text { Characteristics }\end{array}$ & $\begin{array}{c}\text { Total } \\
(n=341)\end{array}$ & $\begin{array}{l}\text { No EACA } \\
\text { Treatment } \\
(n=195)\end{array}$ & $\begin{array}{c}\text { EACA } \\
\text { Treatment } \\
(n=146)\end{array}$ & $\begin{array}{c}\mathrm{p} \\
\text { Value }\end{array}$ \\
\hline $\begin{array}{l}\text { Confirmed aneurysm } \\
\qquad \begin{array}{l}1 \\
>1\end{array}\end{array}$ & $\begin{array}{r}255(74.8) \\
86(25.2)\end{array}$ & $\begin{array}{r}143(73.3) \\
52(26.7)\end{array}$ & $\begin{array}{r}112(76.7) \\
34(23.3)\end{array}$ & 0.477 \\
\hline $\begin{array}{l}\text { Location } \\
\text { Anterior } \\
\text { Posterior }\end{array}$ & $\begin{array}{l}214(62.8) \\
127(37.2)\end{array}$ & $\begin{array}{r}135(69.2) \\
60(30.8)\end{array}$ & $\begin{array}{l}79(54.1) \\
67(45.9)\end{array}$ & 0.004 \\
\hline $\begin{array}{l}\text { Location } \\
\text { Left } \\
\text { Right } \\
\text { Midline }\end{array}$ & $\begin{array}{r}105(30.8) \\
95(27.9) \\
141(41.3)\end{array}$ & $\begin{array}{l}67(34.4) \\
63(32.3) \\
65(33.3)\end{array}$ & $\begin{array}{l}38(26.0) \\
32(21.9) \\
76(52.1)\end{array}$ & 0.002 \\
\hline Size in mm & & & & 0.079 \\
\hline Median & 7 & 7 & 8 & \\
\hline IQR & $5-10$ & $5-9$ & $5-10$ & \\
\hline $\begin{array}{l}\text { Time to intervention } \\
\text { in hrs } \dagger\end{array}$ & & & & $<0.001$ \\
\hline Median & 23 & 19 & 28 & \\
\hline IQR & $14-44$ & $12-38$ & $16-49$ & \\
\hline Hunt \& Hess grade & & & & 0.039 \\
\hline Median & II & II & III & \\
\hline IQR & I-IV & $|-|||$ & I-IV & \\
\hline Fisher grade & & & & 0.984 \\
\hline Median & 3 & 3 & 3 & \\
\hline IQR & $3-4$ & $3-4$ & $3-4$ & \\
\hline
\end{tabular}

* Values represent the number of patients (\%) unless indicated otherwise. Boldface type indicates statistical significance.

† Time to intervention was not available in 27 patients.

\section{Discussion}

To contextualize the results of this study, we reviewed the literature on antifibrinolytic therapy for preventing rehemorrhage after aSAH. Since our study is the first that focuses on EACA treatment in patients undergoing endovascular repair, comparisons are inevitably made with the available literature on surgical repair. Rebleeding after aSAH is thought to depend on the equilibrium between coagulation cascades and fibrinolysis in the aneurysmal microenvironment. ${ }^{15,31,42}$ Antifibrinolytic agents, including EACA, tranexamic acid (TXA), and their equivalents, have been investigated in the prevention of preoperative rebleeding. Antifibrinolytic agents function through competitive inhibition of plasminogen activation, preventing the formation of the proteolytic enzyme plasmin and thereby preventing fibrin degradation within the hemostatic plug. ${ }^{29}$ The role of these agents is not fully established with respect to treatment efficacy and the potential for harm in the form of delayed cerebral ischemia.

\section{Rebleeding Incidence}

The use of antifibrinolytic therapy after aSAH was first proposed in 1968 by Mullan and Dawley. ${ }^{34}$ Based 
TABLE 3. Comparison of aSAH complications and outcomes (univariate analysis)*

\begin{tabular}{|c|c|c|c|c|}
\hline Complications or Outcome & $\begin{array}{c}\text { Total } \\
(\mathrm{n}=341)\end{array}$ & $\begin{array}{l}\text { No EACA } \\
(n=195)\end{array}$ & $\begin{array}{c}\text { EACA } \\
(n=146)\end{array}$ & $\begin{array}{c}\mathrm{p} \\
\text { Value }\end{array}$ \\
\hline $\begin{array}{l}\text { Preinterventional rebleed- } \\
\text { ing }\end{array}$ & $12(3.5)$ & $6(3.1)$ & $6(4.1)$ & 0.609 \\
\hline Vasospasm & $58(17.0)$ & $27(13.8)$ & $31(21.2)$ & 0.072 \\
\hline Clinical stroke & $29(8.5)$ & $14(7.2)$ & $15(10.3)$ & 0.265 \\
\hline Radiological infarction & $51(15.0)$ & $33(16.9)$ & $18(12.3)$ & 0.295 \\
\hline Hydrocephalus & $130(38.1)$ & $72(36.9)$ & $58(39.7)$ & 0.598 \\
\hline Shunt & $49(14.4)$ & $19(9.7)$ & $30(20.5)$ & 0.004 \\
\hline Systemic complications & $94(27.6)$ & $58(29.7)$ & $36(24.7)$ & 0.298 \\
\hline Pulmonary embolism & $3(0.9)$ & $1(0.5)$ & $2(1.4)$ & 0.577 \\
\hline Deep venous thrombosis & $17(5.0)$ & $10(5.1)$ & $7(4.8)$ & 0.970 \\
\hline Cardiac complications & $18(5.3)$ & $15(7.7)$ & $3(2.1)$ & 0.027 \\
\hline Pulmonary edema & $7(2.1)$ & $1(0.5)$ & $6(4.1)$ & 0.045 \\
\hline Respiratory insufficiency & $67(19.7)$ & $43(22.1)$ & $24(16.4)$ & 0.218 \\
\hline Length of stay in days & & & & $<0.001$ \\
\hline Median & 17 & 14 & 19 & \\
\hline IQR & $10-26$ & $10-23$ & $12.5-30$ & \\
\hline $\begin{array}{l}\text { Death during hospitaliza- } \\
\text { tion }\end{array}$ & $46(13.5)$ & $24(12.3)$ & $22(15.1)$ & 0.460 \\
\hline \multicolumn{5}{|l|}{ GOS score } \\
\hline \multicolumn{5}{|l|}{ Time of discharge } \\
\hline Median & 4 & 4 & 4 & \\
\hline IQR & $3-5$ & $3-5$ & $3-5$ & \\
\hline \multicolumn{5}{|l|}{$6 \mathrm{mos}$} \\
\hline Median & 5 & 4 & 5 & \\
\hline IQR & $4-5$ & $4-5$ & $4-5$ & \\
\hline \multicolumn{5}{|l|}{$1 \mathrm{yr}$} \\
\hline Median & 5 & 5 & 5 & \\
\hline IQR & $4-5$ & $4-5$ & $4-5$ & \\
\hline
\end{tabular}

* Values represent the number of patients (\%) unless indicated otherwise. Boldface type indicates statistical significance.

on the management practice of delayed surgical intervention in that era, antifibrinolytic therapy was used for 2-6 weeks. ${ }^{31,34}$ It demonstrated reduced rebleeding ${ }^{11,26,43}$ but an increased incidence of delayed cerebral ischemia. ${ }^{10,27,43}$

Over the past 30 years, the trend for the care of ruptured aneurysms has centered on early intervention, ${ }^{29}$ alleviating the need for prolonged antifibrinolytic therapy. However, there still may be a significant time interval between the onset of hemorrhage and definitive intervention in certain situations, particularly when a patient is transferred to a tertiary care center. During this period, maximizing protection from rebleeding is desirable. The Cochrane database first published a systematic review in 2003, which halted the use of antifibrinolytic therapy due to an overall nonstatistically significant increase in detrimental outcomes from delayed ischemic deficits associated with antifibrinolytic therapy. ${ }^{37}$ This study was updated in 2013 to include an additional study, thereby including
TABLE 4. Multivariate analysis of aSAH complications and outcomes*

\begin{tabular}{lccc}
\hline \multicolumn{1}{c}{ Response Variable } & $\begin{array}{c}\text { OR (EACA } \\
\text { no EACA) }\end{array}$ & $95 \% \mathrm{Cl}$ & $\begin{array}{c}\mathrm{p} \\
\text { Value }\end{array}$ \\
\hline Preinterventional rebleeding & 0.782 & $0.176-3.480$ & 0.747 \\
\hline Vasospasm & 1.314 & $0.705-2.446$ & 0.390 \\
\hline Clinical stroke & 1.877 & $0.826-4.264$ & 0.133 \\
\hline Radiological infarction & 0.656 & $0.331-1.297$ & 0.225 \\
\hline Hydrocephalus & 0.798 & $0.472-1.348$ & 0.398 \\
\hline Shunt & 2.047 & $1.043-4.018$ & $\mathbf{0 . 0 3 7}$ \\
\hline Systemic complications & 0.573 & $0.318-1.032$ & 0.064 \\
\hline Pulmonary embolism & 2.119 & $0.167-26.974$ & 0.563 \\
\hline Deep venous thrombosis & 0.848 & $0.283-2.547$ & 0.770 \\
\hline Cardiac complications & 0.138 & $0.031-0.604$ & $\mathbf{0 . 0 0 9}$ \\
\hline Pulmonary edema† & & & \\
\hline Respiratory insufficiency & 0.471 & $0.239-0.926$ & $\mathbf{0 . 0 2 9}$ \\
\hline Death during hospitalization & 1.152 & $0.560-2.373$ & 0.700 \\
\hline
\end{tabular}

* Boldface type indicates statistical significance.

† No patient remained in the "No EACA" group after excluding the 27 patients in whom time to intervention was not available.

1904 patients in the systematic review, but all 10 analyzed studies had been published prior to 2002. ${ }^{2}$ Importantly, the antifibrinolytic treatment duration for patients in these studies ranged from less than 72 hours up to 6 weeks, with 9 studies using TXA (4-9 g/day) and 1 study using EACA (24 g/day). Despite its interstudy heterogeneity, this metaanalysis revealed a reduced risk of rehemorrhage (risk ratio [RR] $0.65,95 \%$ CI $0.44-0.97$ ) and an increased risk for cerebral ischemia (RR 1.41, 95\% CI 1.04-1.91). The overall results demonstrated no benefit in either mortality or overall outcome. ${ }^{2}$ This lack of disparity in outcomes is thought to represent a balance between the risk of recurrent hemorrhage and the risk of cerebral ischemia.

Our group conducted 2 studies, one with 307 patients and another with 164 patients, to evaluate the utility of a short-term $(<72$-hour) high-dose EACA protocol before early microsurgery. The goal of this protocol was to concomitantly decrease the risk for rehemorrhage while avoiding iatrogenic cerebral ischemia. The results of these 2 studies showed a $1.3 \%$ and $2.8 \%$ incidence of rebleeding and an $8 \%$ and $7.4 \%$ incidence of delayed cerebral deficits. ${ }^{29,30}$ Our previous studies either included historical controls abstracted from previous reports or did not conduct any comparative statistical analysis.

A 505-patient randomized controlled trial using TXA, ${ }^{19}$ a 356-patient retrospective cohort study using EACA, and a 73-patient controlled prospective nonrandomized EACA study, ${ }^{17,41}$ investigated short-term antifibrinolytic therapy. Results of the TXA- and EACA-controlled studies reported significant reduction in the incidence of rebleeding in the treated versus untreated groups: $2.4 \%$ versus $10.8 \%$, and $2.7 \%$ versus $11.4 \%$, respectively. ${ }^{19,41}$ Both of these studies were included in a meta-analysis that examined 17 studies with a total of 2872 patients and represented the only studies available that investigated short-term antifi- 


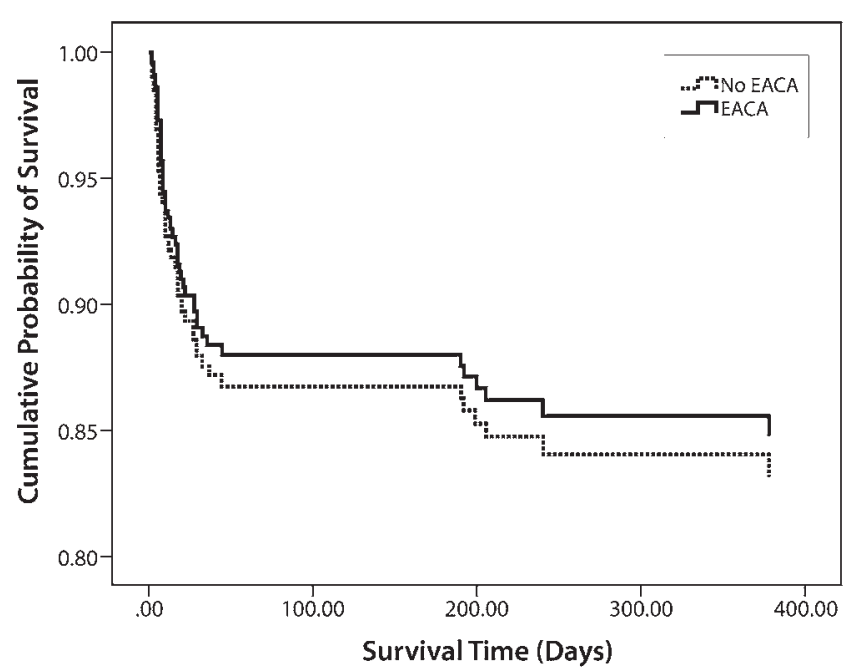

FIG. 1. Survival analysis. Adjusted HR 0.998, 95\% Cl 0.541-1.839; $p=$ 0.994 .

brinolytic therapy. ${ }^{13}$ The results of the meta-analysis further supported the benefits of a short-term ( $<72$ hours) treatment regimen to prevent rebleeding and decrease the risk of poor functional outcome..$^{13}$

As a result of the available data for short-term antifibrinolytic regimen, the American Heart Association/ American Stroke Association published its most recent guidelines stating that short-term $(<72$ hours) antifibrinolytic therapy, in the absence of contraindications, is a reasonable option in the setting of an unavoidable delay in aneurysm obliteration during the interim between clinical presentation and definitive aneurysm repair. ${ }^{6}$ Additional short-term treatment data will be needed to reliably assess the validity of the American Heart Association/American Stroke Association guidelines for modern aSAH management. The previous systematic review by Baharoglu et al. in 2013 on antifibrinolytic therapy for aSAH included only one study analyzing antifibrinolysis with a duration of less than 72 hours, and these studies did not routinely use hypervolemic therapy or calcium channel blockers. ${ }^{2}$

Uniquely, the results of our current study indicate that EACA was not associated with a diminished rate of preinterventional rebleeding in the multivariate analysis for the patients receiving endovascular coiling, $3.1 \%$ and $4.1 \%$ for EACA-treated and untreated cohorts, respectively (OR $0.782,95 \%$ CI $0.176-3.480$ ). This is not consistent with the previous studies at our institution or the others that we have reviewed, keeping in mind that these studies refer to the patients who underwent microsurgery. $17,19,29,30,41$

In our study, multiple variables may have accounted for the lack of efficacy of EACA in decreasing the risk of rebleeding. At our institution, the time from aneurysm rupture to intervention has progressively decreased in the recent years between our current and prior studies. Unfortunately, despite our efforts, we were unable to accurately assess the time of initial hemorrhage to definitive treatment in our patients to objectively measure the effect of this parameter on the current results and compare the findings with our previous studies. Some of our patients were transferred from outside institutions and therefore the exact time from ictus to treatment remains unknown. There is also a notable variation in the literature regarding rebleeding rates for the groups assigned to early intervention. A literature review by Starke and Connolly reported that the likely percentage of rebleed rates is between $5.3 \%$ and $11.4 \%$, with most rebleeds occurring within 6 hours of the initial hemorrhage. ${ }^{40}$

A more recent study of 293 patients confirmed rebleed rates of $12 \%$ with an additional $4 \%$ having possible rebleeds within a median time from initial hemorrhage to rebleed of 3 hours. ${ }^{14} \mathrm{~A}$ progressive decrease in the time from diagnosis until intervention is likely to be different for the patients in the endovascular and microsurgical group, similar to the findings in the ISAT (International Subarachnoid Aneurysm Trial) and BRAT (Barrow Ruptured Aneurysm Trial) studies. ${ }^{31,33}$ This can potentially explain the lack of reduction in the rehemorrhage rates in our cohort promptly treated via endovascular coiling. This phenomenon emphasizes an important point about evaluation of antifibrinolytic efficacy research-when comparing study results for rebleed rates and outcome scores, the variable of endovascular versus microsurgical intervention can confound comparison analysis. Unfortunately, a reliable assessment of time of initial hemorrhage to treatment can be problematic because of the high number of patients undergoing interhospital transfers. The type of antifibrinolytic agent used is another important variable to consider when comparing study results.

\section{Differences in Antifibrinolytic Agents}

Although EACA was used in our institution's previous studies, this antifibrinolytic agent was used in only 1 of the 10 studies included in the Cochrane systematic review. ${ }^{2}$ The other 9 studies in that review used TXA. This difference may have contributed to differing results with respect to the prevention of rebleeding. TXA and EACA differ in plasminogen/plasmin binding affinity; TXA has 6-10 times greater affinity. ${ }^{8}$ However, placebo-controlled studies during cardiac surgery have demonstrated comparable efficacy in antifibrinolytic activity between TXA and EACA. . $^{1620}$

\section{Antifibrinolytic Agent Dosing and Thrombotic Risk}

Historically, studies found in the literature described a 24- to 48-hour delay in the onset of EACA effectiveness, ${ }^{4,12,26}$ which was later attributed to the lack of a loading dose. ${ }^{27,36}$ Our study in 1997 monitored serum levels of EACA. We found that therapeutic levels were achieved within 24 hours through a bolus dose followed by subsequent continuous intravenous infusion..$^{30}$ Starke et al. used a 4-g loading dose with a $1-\mathrm{g} / \mathrm{hr}$ maintenance infusion and demonstrated a beneficial impact on rebleeding rates. ${ }^{41}$ The dosage regimen used in our study (a 5- to 10-g bolus dose with subsequent $2-\mathrm{g} / \mathrm{hr}$ infusion) should allow for similar therapeutic efficacy. Our institution's treatment regimen differs slightly from those of other centers in that an antifibrinolytic agent (EACA) is routinely infused until the patient is transported to the procedure 
or occasionally until the aneurysm is excluded via coil embolization. This is in contrast to the routine at other institutions, which stop antifibrinolytic treatment at least 4 hours prior to the procedure because of the perceived thrombotic risk. ${ }^{17,41}$

Aneurysmal SAH potentially creates a prothrombotic and proinflammatory state as indicated by serum markers, such as platelet count, leukocyte count, erythrocyte sedimentation rate, and C-reactive protein levels. ${ }^{25}$ In addition, thrombotic risks are reported to be higher with endovascular treatment compared with microsurgery. ${ }^{32,35}$ There is a concern that the combination of antifibrinolytic treatment in the setting of endovascular coiling and aSAH could potentiate thrombotic risk. In a retrospective study of 83 patients, with short-term $(<72$ hours $)$ EACA therapy in angiographically negative $\mathrm{SAH}$, Hui et al. reported no increase in thrombotic complications, including deep venous thrombosis $(p=0.65)$, pulmonary embolism ( $p$ $=1.0)$, delayed cerebral ischemia $(\mathrm{p}=1.0)$, and myocardial infarction $(\mathrm{p}=1.0) .{ }^{21}$ This supports a benign adverse thromboembolic effect profile for short-term EACA use for prevention of rebleeding. However, a nonrandomized prospective controlled study by Starke et al. demonstrated an 8-fold increased risk of deep venous thrombosis with a similar short-term EACA regimen, but the authors failed to show an increased risk for pulmonary embolism or delayed cerebral ischemia. ${ }^{41}$

\section{Long-Term Outcomes}

Despite the potential efficacy of antifibrinolytic therapy in reducing the risk of rebleeding, the studies on shortterm administration of the drug have not demonstrated a

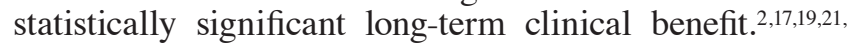
${ }^{39,41}$ This is consistent with our findings. However, a pooled analysis by Starke et al. ${ }^{41}$ with study data from Hillman et al. ${ }^{19}$ showed a $9 \%$ increase in favorable 3-month modified Rankin Scale score (0-3). Earlier studies on prolonged use of antifibrinolytic agents suggested that an increase in risk of delayed cerebral ischemia offsets the benefits from reduction in the rate of rebleeding; ${ }^{2}$ however, in our study, neither the risk of rebleeding nor ischemia was significantly different between the 2 groups. Similar recent studies investigating short-term antifibrinolytic therapy have also failed to demonstrate a statistically significant increased risk of ischemia compared with controls. ${ }^{19,21,39,41}$ Therefore, the idea that the lack of improved outcomes with antifibrinolytic therapy is explained by an increased rate of ischemia is not supported by recent studies involving shortterm antifibrinolytic therapy. To determine the efficacy of antifibrinolytic therapy in improving long-term outcomes, additional randomized controlled trials are required.

\section{Length of Stay}

Our data indicate that the median length of hospital stay for the EACA-treated patients was significantly longer than that for the control patients, 19 days and 14 days, respectively $(\mathrm{p}<0.001)$. This difference is not likely related to EACA treatment, but rather correlates with the degree of asymmetry in clinical condition between the groups, with the EACA group including patients who presented in a worse clinical status.

\section{Hydrocephalus Incidence}

Chronic hydrocephalus is a known sequela of aSAH, with rates ranging from $20 \%$ to $63.4 \%{ }^{18,28}$ and risk factors including aneurysm location and volume of hemorrhage. ${ }^{1}$ In our study, the rates for ventricular shunt placement in the EACA versus no-EACA cohorts were $20.5 \%$ and $9.7 \%$ $(\mathrm{p}=0.004)$, respectively.

The Cochrane systematic review of 10 studies included 5 that reported the incidence of hydrocephalus, all of which used longer courses of antifibrinolytic agents ( $>72$ hours). ${ }^{2}$ Compilation of this data determined that antifibrinolytic use was not associated with an increase the incidence of hydrocephalus (RR 1.11, 95\% CI 0.90-1.36). ${ }^{2}$ Harrigan et al. found no statistical difference in the incidence of either ventriculostomy or shunt placement when comparing microsurgical and endovascular intervention for patients with aSAH. ${ }^{17}$ The data from our study, which exclusively evaluated an endovascularly treated population, demonstrated a significant correlation between EACA treatment and placement of a shunt but not the overall incidence of hydrocephalus. It should be noted that the EACA group had a higher incidence of midline aneurysms and a higher initial Hunt and Hess score, which may also have contributed to the need for a shunt. Also, we did not specifically review the incidence of intraventricular hemorrhage in this group due to the unavailability of the data.

\section{Limitations}

This study is primarily limited by being a retrospective cohort from a single institution. We tried to partially overcome this limitation by expanding the study over a 12-year period, yet prospective multicenter studies would eliminate this limitation and increase the patient sample size. The relatively small sample size in this study may overlook the small differences in the rate of preintervention rebleeding. Finally, since the patients were not randomly assigned to treated and untreated groups, there is the possibility of selection bias. All of these limitations would be ideally addressed in well-designed prospective multicenter studies.

\section{Conclusions}

To our knowledge, this is the first study that specifically assesses short-term EACA use in aSAH patients undergoing endovascular treatment. We were unable to detect a significant difference in the risk of cerebral ischemia related to the use of EACA. However, we also did not detect a difference in preintervention rehemorrhage rates. To better define the role of EACA in aSAH and to develop a robust clinical guideline for the use of antifibrinolytic therapies, a randomized placebo-controlled trial is necessary.

\section{References}

1. Bae IS, Yi HJ, Choi KS, Chun HJ: Comparison of Incidence and Risk Factors for Shunt-dependent Hydrocephalus in Aneurysmal Subarachnoid Hemorrhage Patients. J Cerebrovasc Endovasc Neurosurg 16:78-84, 2014

2. Baharoglu MI, Germans MR, Rinkel GJ, Algra A, Vermeulen M, van Gijn J, et al: Antifibrinolytic therapy for aneurys- 
mal subarachnoid haemorrhage. Cochrane Database Syst Rev 8:CD001245, 2013

3. Broderick JP, Brott TG, Duldner JE, Tomsick T, Leach A: Initial and recurrent bleeding are the major causes of death following subarachnoid hemorrhage. Stroke 25:1342-1347, 1994

4. Burchiel KJ, Hoffman JM, Bakay RA: Quantitative determination of plasma fibrinolytic activity in patients with ruptured intracranial aneurysms who are receiving epsilon-aminocaproic acid: relationship of possible complications of therapy to the degree of fibrinolytic inhibition. Neurosurgery 14:57-63, 1984

5. Cha KC, Kim JH, Kang HI, Moon BG, Lee SJ, Kim JS: Aneurysmal rebleeding: factors associated with clinical outcome in the rebleeding patients. J Korean Neurosurg Soc 47:119-123, 2010

6. Connolly ES Jr, Rabinstein AA, Carhuapoma JR, Derdeyn CP, Dion J, Higashida RT, et al: Guidelines for the management of aneurysmal subarachnoid hemorrhage: a guideline for healthcare professionals from the American Heart Association/ American Stroke Association. Stroke 43:1711-1737, 2012

7. Cross DT III, Tirschwell DL, Clark MA, Tuden D, Derdeyn $\mathrm{CP}$, Moran CJ, et al: Mortality rates after subarachnoid hemorrhage: variations according to hospital case volume in 18 states. J Neurosurg 99:810-817, 2003

8. Dunn CJ, Goa KL: Tranexamic acid: a review of its use in surgery and other indications. Drugs 57:1005-1032, 1999

9. Fisher CM, Kistler JP, Davis JM: Relation of cerebral vasospasm to subarachnoid hemorrhage visualized by computerized tomographic scanning. Neurosurgery 6:1-9, 1980

10. Fodstad H, Forssell A, Liliequist B, Schannong M: Antifibrinolysis with tranexamic acid in aneurysmal subarachnoid hemorrhage: a consecutive controlled clinical trial. Neurosurgery 8:158-165, 1981

11. Fodstad H, Liliequist B, Schannong M, Thulin CA: Tranexamic acid in the preoperative management of ruptured intracranial aneurysms. Surg Neurol 10:9-15, 1978

12. Fodstad H, Pilbrant A, Schannong M, Strömberg S: Determination of tranexamic acid (AMCA) and fibrin/fibrinogen degradation products in cerebrospinal fluid after aneurysmal subarachnoid haemorrhage. Acta Neurochir (Wien) 58:1-13, 1981

13. Gaberel T, Magheru C, Emery E, Derlon JM: Antifibrinolytic therapy in the management of aneurismal subarachnoid hemorrhage revisited. A meta-analysis. Acta Neurochir (Wien) 154:1-9, 2012

14. Germans MR, Coert BA, Vandertop WP, Verbaan D: Time intervals from subarachnoid hemorrhage to rebleed. J Neurol 261:1425-1431, 2014

15. Gibbs JR, O'Gorman P: Fibrinolysis in subarachnoid haemorrhage. Postgrad Med J 43:779-784, 1967

16. Hardy JF, Bélisle S, Dupont C, Harel F, Robitaille D, Roy M, et al: Prophylactic tranexamic acid and epsilon-aminocaproic acid for primary myocardial revascularization. Ann Thorac Surg 65:371-376, 1998

17. Harrigan MR, Rajneesh KF, Ardelt AA, Fisher WS III: Short-term antifibrinolytic therapy before early aneurysm treatment in subarachnoid hemorrhage: effects on rehemorrhage, cerebral ischemia, and hydrocephalus. Neurosurgery 67:935-940, 2010

18. Hasan D, Vermeulen M, Wijdicks EF, Hijdra A, van Gijn $\mathrm{J}$ : Management problems in acute hydrocephalus after subarachnoid hemorrhage. Stroke 20:747-753, 1989

19. Hillman J, Fridriksson S, Nilsson O, Yu Z, Saveland H, Jakobsson KE: Immediate administration of tranexamic acid and reduced incidence of early rebleeding after aneurysmal subarachnoid hemorrhage: a prospective randomized study. J Neurosurg 97:771-778, 2002

20. Horrow JC, Van Riper DF, Strong MD, Brodsky I, Parmet JL:
Hemostatic effects of tranexamic acid and desmopressin during cardiac surgery. Circulation 84:2063-2070, 1991

21. Hui FK, Schuette AJ, Lieber M, Spiotta AM, Moskowitz SI, Barrow DL, et al: $\varepsilon$-Aminocaproic acid in angiographically negative subarachnoid hemorrhage patients is safe: a retrospective review of 83 consecutive patients. Neurosurgery 70:702-706, 2012

22. Hunt WE, Hess RM: Surgical risk as related to time of intervention in the repair of intracranial aneurysms. J Neurosurg 28:14-20, 1968

23. Hunt WE, Meagher JN, Hess RM: Intracranial aneurysm. A nine-year study. Ohio State Med J 62:1168-1171, 1966

24. Josephson SA, Douglas VC, Lawton MT, English JD, Smith WS, Ko NU: Improvement in intensive care unit outcomes in patients with subarachnoid hemorrhage after initiation of neurointensivist co-management. J Neurosurg 112:626-630, 2010

25. Kasius KM, Frijns CJ, Algra A, Rinkel GJ: Association of platelet and leukocyte counts with delayed cerebral ischemia in aneurysmal subarachnoid hemorrhage. Cerebrovasc Dis 29:576-583, 2010

26. Kassell NF, Torner JC: Aneurysmal rebleeding: a preliminary report from the Cooperative Aneurysm Study. Neurosurgery 13:479-481, 1983

27. Kassell NF, Torner JC, Adams HP Jr: Antifibrinolytic therapy in the acute period following aneurysmal subarachnoid hemorrhage. Preliminary observations from the Cooperative Aneurysm Study. J Neurosurg 61:225-230, 1984

28. Klopfenstein JD, Kim LJ, Feiz-Erfan I, Hott JS, Goslar P, Zabramski JM, et al: Comparison of rapid and gradual weaning from external ventricular drainage in patients with aneurysmal subarachnoid hemorrhage: a prospective randomized trial. J Neurosurg 100:225-229, 2004

29. Leipzig TJ, Horner TG, Payner TD, Redelman K: Protection from rebleeding before early aneurysm surgery. Semin Neurosurg 11:3-16, 2000

30. Leipzig TJ, Redelman K, Horner TG: Reducing the risk of rebleeding before early aneurysm surgery: a possible role for antifibrinolytic therapy. J Neurosurg 86:220-225, 1997

31. McDougall CG, Spetzler RF, Zabramski JM, Partovi S, Hills NK, Nakaji P, et al: The Barrow Ruptured Aneurysm Trial. J Neurosurg 116:135-144, 2012

32. Molyneux A, Kerr R, Stratton I, Sandercock P, Clarke M, Shrimpton J, et al: International Subarachnoid Aneurysm Trial (ISAT) of neurosurgical clipping versus endovascular coiling in 2143 patients with ruptured intracranial aneurysms: a randomised trial. Lancet 360:1267-1274, 2002

33. Molyneux AJ, Kerr RS, Yu LM, Clarke M, Sneade M, Yarnold JA, et al: International Subarachnoid Aneurysm Trial (ISAT) of neurosurgical clipping versus endovascular coiling in 2143 patients with ruptured intracranial aneurysms: a randomised comparison of effects on survival, dependency, seizures, rebleeding, subgroups, and aneurysm occlusion. Lancet 366:809-817, 2005

34. Mullan S, Dawley J: Antifibrinolytic therapy for intracranial aneurysms. J Neurosurg 28:21-23, 1968

35. Naidech AM, Rosenberg NF, Maas MB, Bendok BR, Batjer $\mathrm{HH}$, Nemeth AJ: Predictors of hemorrhage volume and disability after perimesencephalic subarachnoid hemorrhage. Neurology 78:811-815, 2012

36. Pechet L: Fibrinolysis. N Engl J Med 273:1024-1034, 1965

37. Roos YB, Rinkel GJ, Vermeulen M, Algra A, van Gijn J: Antifibrinolytic therapy for aneurysmal subarachnoid haemorrhage. Cochrane Database Syst Rev (2):CD001245, 2003

38. Rosengart AJ, Schultheiss KE, Tolentino J, Macdonald RL: Prognostic factors for outcome in patients with aneurysmal subarachnoid hemorrhage. Stroke 38:2315-2321, 2007

39. Schuette AJ, Hui FK, Obuchowski NA, Walkup RR, Cawley $\mathrm{CM}$, Barrow DL, et al: An examination of aneurysm rerup- 
ture rates with epsilon aminocaproic acid. Neurocrit Care 19:48-55, 2013

40. Starke RM, Connolly ES Jr: Rebleeding after aneurysmal subarachnoid hemorrhage. Neurocrit Care 15:241-246, 2011

41. Starke RM, Kim GH, Fernandez A, Komotar RJ, Hickman ZL, Otten ML, et al: Impact of a protocol for acute antifibrinolytic therapy on aneurysm rebleeding after subarachnoid hemorrhage. Stroke 39:2617-2621, 2008

42. Tsementzis SA, Hitchcock ER, Meyer CH: Benefits and risks of antifibrinolytic therapy in the management of ruptured intracranial aneurysms. A double-blind placebo-controlled study. Acta Neurochir (Wien) 102:1-10, 1990

43. Vermeulen M, Lindsay KW, Murray GD, Cheah F, Hijdra A, Muizelaar JP, et al: Antifibrinolytic treatment in subarachnoid hemorrhage. N Engl J Med 311:432-437, 1984

44. Winn HR, Richardson AE, Jane JA: The long-term prognosis in untreated cerebral aneurysms: I. The incidence of late hemorrhage in cerebral aneurysm: a 10-year evaluation of 364 patients. Ann Neurol 1:358-370, 1977

\section{Disclosures}

Dr. Shah reports that he is a consultant for Stryker.

\section{Author Contributions}

Conception and design: Cohen-Gadol, Malekpour, Bohnstedt, Radmand, Sethia, Hendricks, Leipzig, Payner, Shah, Scott, DeNardo. Acquisition of data: Cohen-Gadol, Malekpour, Kulwin, Radmand, Sethia, Hendricks, Leipzig, Payner, Shah, Scott, DeNardo, Sahlein. Analysis and interpretation of data: all authors. Drafting the article: Cohen-Gadol, Malekpour, Bohnstedt, Radmand, Sethia, Mendenhall, Weyhenmeyer, Hendricks, Leipzig, Payner, Shah, Scott, DeNardo, Sahlein. Critically revising the article: all authors. Reviewed submitted version of manuscript: all authors. Approved the final version of the manuscript on behalf of all authors: Cohen-Gadol.

Statistical analysis: Cohen-Gadol, Malekpour, Radmand, Sethia, Mendenhall, Weyhenmeyer, Hendricks, Scott, DeNardo, Sahlein. Administrative/technical/material support: Cohen-Gadol, Malekpour, Mendenhall, Weyhenmeyer, Leipzig, DeNardo. Study supervision: Cohen-Gadol, Malekpour.

\section{Correspondence}

Aaron A. Cohen-Gadol, Goodman Campbell Brain and Spine, Indiana University, Department of Neurological Surgery, 355 W 16th St., Ste. \#5100, Indianapolis, IN 46202. email: acohenmd@ gmail.com. 